\title{
Full-Duplex UAV Relay Positioning for Vehicular Communications with Underlay V2V Links
}

\author{
Pouya Pourbaba, K. B. Shashika Manosha, Samad Ali, and Nandana Rajatheva \\ Center for Wireless Communications (CWC), University of Oulu, Oulu, Finland \\ pouya.pourbaba@student.oulu.fi, \{manosha.kapuruhamybadalge, samad.ali, nandana.rajatheva\}@oulu.fi
}

\begin{abstract}
The unmanned aerial vehicles (UAVs) can be deployed as aerial base stations or wireless relays to increase the capacity of wireless networks. In this paper, the positioning of a full-duplex (FD) UAV as a relay to provide coverage for an FD vehicular network is investigated. In particular, given a configuration of predefined locations for the UAV, and the position of the vehicular users on the ground, a novel algorithm is proposed to find the position for the UAV to satisfy the quality of service $(\mathrm{QOS})$ requirements of the vehicles in the network. The positioning problem is formulated as an $\ell_{0}$ minimization which is non-combinatorial , NP-hard, and finding a globally optimal solution for this problem has exponential complexity. Hence, we have approximated the nonconvex problem using $\ell_{1}$ norm and proposed a suboptimal algorithm to solve it. Simulation results show that by using the proposed approach the number of times that UAV can satisfy the SINR constraints increases by approximately $10 \%$ compared to a baseline scenario in which the UAV has a fixed location.
\end{abstract}

Index Terms-Full-duplex UAV, Relaying, V2V Communications, Convex Optimization

\section{INTRODUCTION}

Autonomous vehicles are expected to increase the efficiency and safety of future transport systems. Recently, there has been a surge of interest in developing intelligent transport systems (ITS) and addressing the associated problems [1]. For example, the safety is a crucial issue especially in the case of self-driving cars which ITS aims to address by concepts such as vehicle-to-vehicle $(\mathrm{V} 2 \mathrm{~V})$ communications and vehicle-toinfrastructure (V2I) communications [2], [3]. Another crucial emerging phenomenon which has attracted a lot of attention is the use of unmanned aerial vehicles (UAVs) as base stations (BSs) in emergencies where it is required to recover the wireless network due to damages from natural disasters such as flood or earthquake [4]. Moreover, the UAVs can be deployed as wireless relays to enhance the communication between wireless devices [5]. However, UAV communications and networking for vehicular networks faces serious challenges such as the high mobility of vehicles and air to ground channel modeling [6]-[8]. Moreover, it is necessary to design power efficient methods due to the limited capacity of the batteries in the UAVs.

To address the aforementioned challenges, the authors in [9] propose a new framework for locating and modeling several UAVs in a 3D space, where these UAVs are utilized as aerial BSs to collect data from the IoT devices on the ground. The location of the UAV and the uplink power are determined such that the total transmit power of the devices in the network is minimized subject to their SINR constraints. The deployment of a UAV as an aerial BS which is required to provide wireless communication for a geographical area including device-todevice (D2D) communication network is studied in [7]. It is shown that for different D2D user densities, the UAV can be placed in an optimal height to maximize the system sum-rate and the coverage probability.

Vehicles are expected to be equipped with short-range communication technologies to enable the operative awareness or beaconing where vehicles broadcast their status to the surroundings. Two standards that allow direct $\mathrm{V} 2 \mathrm{~V}$ communication are IEEE 802.11p and longterm evolution V2V (LTE$\mathrm{V} 2 \mathrm{~V})$ [10]. The performance of the IEEE802.11ad medium access control (MAC) and beamforming mechanism are evaluated in [11], where it is shown that IEEE 802.11ad faces serious challenges and some changes are required in order to be able to satisfy the high-bandwidth requirements of vehicular communications. In [12] full duplex radios are porposed to be used in V2V communications since FD radios can achieve up to two times the rate of a conventional half duplex link [13]. The mmWave communication is proposed as another solution for high bandwidth requirement of connected vehicles in [14]. Autonomous vehicles require a large number of sensors to be mounted on them to get information from the surroundings to model the environment around the vehicle. Because of the huge amount of data that autonomous vehicles generate, very high rates are required to transmit the generated data. However, the existing solutions such as $4 \mathrm{G}$ and dedicated short range communications (DSRC) cannot meet the high data rate requirement of the autonomous vehicles. Therefore, novel solutions are required for autonomous vehicular communication systems [14]. With advances in UAV technologies, there has been a surge of interest in using UAVs to address some of the challenges of vehicular communications [8], [15].

A new UAV-assisted data dissemination scheduling strategy in VANETs is proposed in [15] where cooperative data dissemination is used to overcome the limited connection time of the communication links. The authors propose a recursive least square (RLS) algorithm to predict the motion of the vehicles. Moreover, the use of UAVs as assistants for spreading information in vehicular networks is discussed in [8] where the vehicles are grouped in clusters. The UAV is communicating with the head of the cluster, which, decreases the number of links required for the ground users to communicate with 


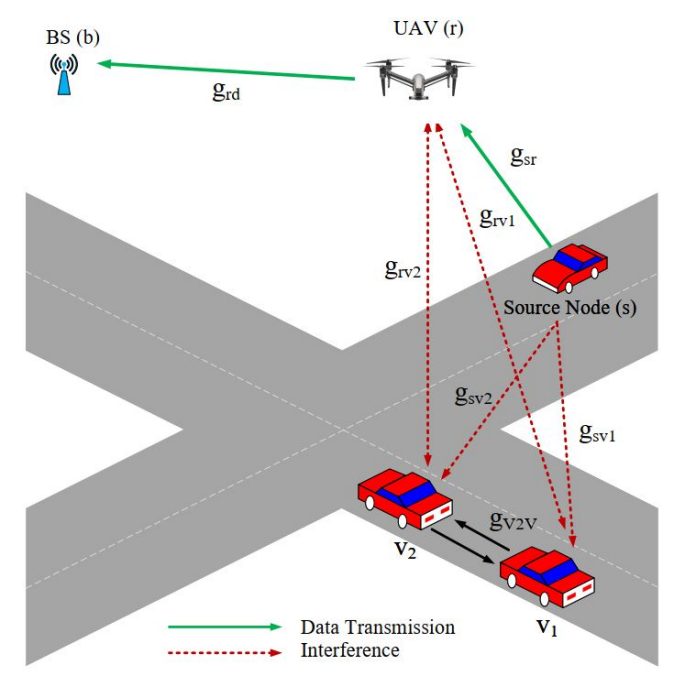

(a) System model

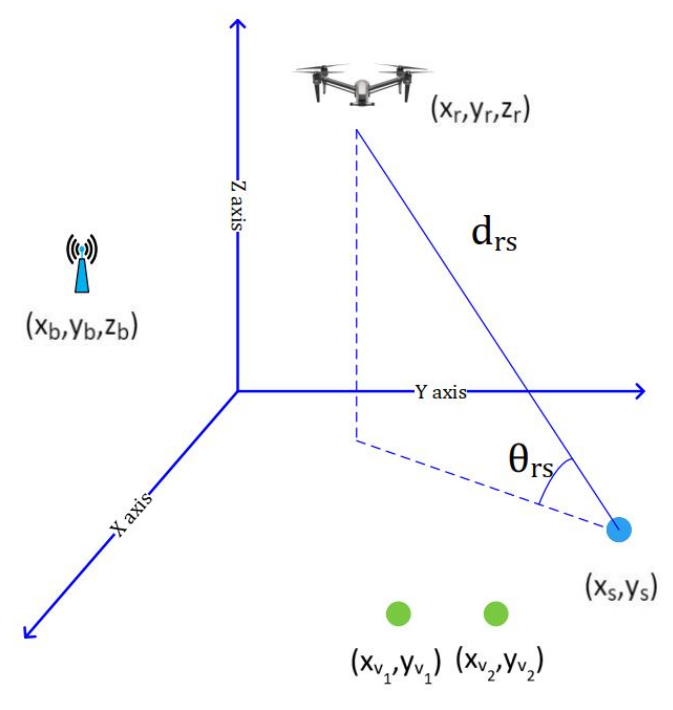

(b) Coordinate system

Figure 1: System model and the coordinate system

each other. Therefore, the interference is reduced and the communication links become more reliable due to the transmit diversity that is introduced by the UAV.

However, to the best of our knowledge, there has been no prior work that investigates the FD UAV relaying in vehicular networks. The main contribution of this paper therefore is to propose a novel method for positioning a UAV which is operating as a wireless relay. First, we define a set of locations that the relay can accommodate and operate at. Next, using $\ell_{0}$-norm we formulate a minimization problem for positioning the UAV such that it can satisfy the QoS requirements for the vehicular network. However, $\ell_{0}$ minimization problem is NP-hard and non-ombinatorial and finding a globally optimal solution requires exponential complexity. Therefore, we relax all the $\ell_{0}$ functions with their natural $\ell_{1}$-norm approximation and convert it into a convex optimization problem. Additionally, the proposed method will find the optimal height for the UAV to operate efficiently.

The rest of the paper is organized as follows. Section II presents the system model describing the air to ground and V2V channel model. In Section III, we formulate the UAV positioning as an $\ell_{1}$ minimization problem. Section IV presents the simulation results, and the conclusions are drawn in section $\mathrm{V}$.

\section{System Model}

Consider a network with a BS, a vehicle as the source node that is communicating with the BS through a UAV relay, and a pair of vehicles which communicate directly with each other through a V2V link. As depicted in Fig. 1(a) the vehicle communicating with the BS is the source node and it is denoted as $s$, the UAV operating as a relay is shown as $r$, the $\mathrm{BS}$ is denoted as the node $b$, and the $\mathrm{V} 2 \mathrm{~V}$ vehicles communicating with each other are shown as $v_{1}$ and $v_{2}$. We assume that the relay and the $\mathrm{V} 2 \mathrm{~V}$ vehicles are operating in a full-duplex mode using the same radio resources, i.e., they are able to transmit and receive signals simultaneously on the same frequency band. Furthermore, we assume that the BS is located far apart, and hence, it is not possible to establish a direct link between the BS and the vehicle. Therefore, the relay is required to assist the source node to communicate with the BS. We denote all the ground devices in the system by the set $\mathcal{D}=\left\{s, b, v_{1}, v_{2}\right\}$. The coordinates of $i$ th device where $i \in \mathcal{D}$ is given by $\left(x_{i}, y_{i}, z_{i}\right)$, and the coordinates of the relay are shown as $\left(x_{r}, y_{r}, z_{r}\right)$. Furthermore, we consider that the locations of the devices are known to a central controller that finds the optimal location of the UAV. Fig. 1(b) illustrates the coordinate system for this setup, where the middle of the junction in Fig. 1(a) is considered as the origin of the coordinate system.

\section{A. V2V Channel Model}

The path loss for vehicular communications is considered to follow the dual-slope model [16] which is given by

$P L(d)= \begin{cases}P L_{0}+10 n_{1} \log _{10}\left(d / d_{0}\right)+X_{\sigma}, & \text { if } d_{0} \leq d \leq d_{b} \\ P L_{0}+10 n_{1} \log _{10}\left(d_{b} / d_{0}\right) & \\ +10 n_{2} \log _{10}\left(d / d_{b}\right)+X_{\sigma}, & \text { if } d \geq d_{b},\end{cases}$

where $d$ is the distance between the transmitter and the receiver, $d_{0}$ is the reference distance, $P L_{0}$ is the path loss at the reference distance, $X_{\sigma}$ is a zero-mean normally distributed random variable with standard deviation of $\sigma$. The notation $d_{b}$ denotes the breakpoint distance where the first Fresnel zone touches the ground, $n_{1}$ is the path loss exponent until the 
distance $d_{b}$, and $n_{2}$ is the path loss exponent for the distances after $d_{b}$. The breakpoint distance is defined as

$$
d_{b}=\frac{4 h_{T X} h_{R X}-\frac{\lambda^{2}}{4}}{\lambda}
$$

where $h_{T X}$ and $h_{R X}$ are the transmitter and the receiver heights, respectively, and $\lambda$ is the wavelength.

\section{B. Air to Ground Channel Model}

In the air-to-ground channel model there are two main groups of signals received in the receiver, the first group is the line of sight (LoS) and the second group is the non-line of sight (NLoS) [4]. The occurrence probabilities of the LoS and NLoS links are a function of the environment and the elevation angle between the UAV and the ground user. The parameters defining these probabilities are the average number of the buildings per square kilometer, distribution of the heights of the buildings, and the ratio of the area with buildings to the whole area. The path loss for the LoS and NLoS components can be calculated as a function of the distance between the relay and the ground devices. The following are the path loss equations for the LoS and NLoS links [17]:

$$
\begin{gathered}
L_{L o S}(d B)=\eta_{L o S}\left(\frac{4 \pi f_{c} d_{i}}{c}\right)^{\mu}, \\
L_{N L o S}(d B)=\eta_{N L o S}\left(\frac{4 \pi f_{c} d_{i}}{c}\right)^{\mu},
\end{gathered}
$$

where $\eta_{N L o S}$ and $\eta_{L o S}\left(\eta_{N L o S}>\eta_{L o S}>1\right)$ are the excessive path loss coefficients which are defined according to the propagation group and the physical environment [17], $c$ is the speed of the light, $f_{c}$ is the carrier frequency, $\mu$ is the path loss exponent, and $d_{i}$ is the distance between the relay and the user $i \in D$ on the ground and is calculated by

$$
d_{i}=\sqrt{\left(x_{r}-x_{i}\right)^{2}+\left(y_{r}-y_{i}\right)^{2}+\left(z_{r}-z_{i}\right)^{2}} .
$$

As discussed before, the LoS and NLoS links have their own probabilities of occurrence which depend on the environment characteristics and according to [17] they can be expressed as

$$
\begin{gathered}
P(L o S)=\frac{1}{1+\alpha \exp \left(-\beta\left[\frac{180}{\pi} \theta_{i}-\alpha\right]\right)}, \\
P(N L o S)=1-P(L o S),
\end{gathered}
$$

where $\alpha$ and $\beta$ are constants depending on the type of the environment, $\theta_{i}$ is the elevation angle between the UAV and the ground user $i \in \mathcal{D}, \theta_{i}=\frac{180}{\pi} \times \arcsin \left(\frac{h_{i}}{d_{i}}\right)$, where $h_{i}$ is the vertical distance between the relay and the node $i \in \mathcal{D}$. The following equation denotes the average path loss between the ground user $i$ and the relay [18]:

$L=P(L o S) \times \eta_{L o S}\left(\frac{4 \pi f_{c} d_{i}}{c}\right)^{\mu}+P(N L o S) \times \eta_{N L o S}\left(\frac{4 \pi f_{c} d_{i}}{c}\right)^{\mu}$.

The SINR of the link between the source and the relay is given by

$$
\gamma_{s r}=\frac{p_{s} g_{s r}}{N_{0}+I_{r}+\sum_{j=1}^{2} p_{v_{j}} g_{r v_{j}}}
$$

where $N_{0}$ is the additive white Gaussian noise, $p_{s}$ is the transmit power of the source, $g_{s r}$ is the channel gain between the source and the relay, $I_{r}$ is the residual of the selfinterference (SI) [6]. The residual of SI is defined as $I_{r}=\delta p_{r}$ where $\delta$ depends on the SI cancellation method. The term $\sum_{j=1}^{2} p_{v_{j}} g_{r v_{j}}$ is the total interference from the full-duplex connected vehicles, $p_{v_{j}}$ is the transmit power of the $j^{\text {th }}$ vehicle in the full-duplex link and $g_{r v_{j}}$ is the channel gain between $j$ th vehicle and the relay. The SINR of the FD V2V link can be written as

$$
\gamma_{v_{j}}=\frac{p_{v_{k}} g_{V 2 V}}{N_{0}+I_{v_{j}}+p_{s} g_{s v_{j}}+p_{r} g_{r v_{j}}} \quad j, k \in\{1,2\}, j \neq k,
$$

where $p_{v_{k}}$ is the power of the signal transmitted from the vehicle $v_{k}, g_{V 2 V}$ is the channel gain between the two vehicles, $I_{v_{j}}$ is the residual of the SI, $p_{s} g_{s v_{j}}$ is the interference coming from the source node to the $j$ th $\mathrm{V} 2 \mathrm{~V}$ user, $p_{r} g_{r v_{j}}$ is the interference coming from the relay to the $j$ th $\mathrm{V} 2 \mathrm{~V}$ user. The SNR of the link between the $r$ and $b$ is calculated by

$$
\gamma_{r b}=\frac{p_{r} g_{r b}}{N_{0}},
$$

where $p_{r}$ is the transmit power of the relay, and $g_{r b}$ is the channel gain between the $r$ and the $b$. Since the interference generated by other links can be neglected as we assumed the destination node is located far apart from other links for the link between $r$ and $b$ we have SNR instead of SINR.

\section{PROBLEM FORMULATION AND SOLUTION APPROACH}

In this section, we first formulate the problem of UAV placement. This problem is NP-hard and nonconvex. Therefore, we approximate the original problem with a convex optimization problem and propose a suboptimal method to solve the approximated problem.

\section{A. Problem formulation}

Let us first define the following set of notations. Let $l_{i} \in \mathbb{R}^{3}$ be the $i$ th location that the relay can operate at, where the first, second, and the third elements of $l_{i}$ are $\mathrm{x}, \mathrm{y}$, and $\mathrm{z}$ coordinates, respectively. This matrix is expressed as

$$
\mathbf{L}_{r}=\left[\begin{array}{ccc}
x_{r_{1}} & y_{r_{1}} & z_{r_{1}} \\
x_{r_{2}} & y_{r_{2}} & z_{r_{2}} \\
\vdots & \vdots & \vdots \\
x_{r_{l}} & y_{r_{l}} & z_{r_{l}}
\end{array}\right]
$$

Next, we define the received signal power $\mathbf{s}_{s r} \in \mathbb{R}^{L}$ at each location of the $r$ from $s$. This vector can be expressed as

$$
\mathbf{s}_{s r}=p_{s} \mathbf{g}_{s r} .
$$

where, $p_{s}$ is the transmit power of the $s$ and $\mathbf{g}_{s r} \in \mathbb{R}^{L}$ is channel gain vector for the links between $s$ and each of the predefined locations for $r$. Similarly, we define the interference signal powers received at each location for the relay from the $\mathrm{V} 2 \mathrm{~V}$ vehicles as $\mathbf{s}_{v_{1} r} \in \mathbb{R}^{L}$ and $\mathbf{s}_{v_{2} r} \in \mathbb{R}^{L}$. These vectors are expressed as

$$
\mathbf{s}_{v_{1} r}=p_{v_{1}} \mathbf{g}_{v_{1} r}
$$




$$
\mathbf{s}_{v_{2} r}=p_{v_{2}} \mathbf{g}_{v_{2} r}
$$

where, $p_{v_{1}}$ and $p_{v_{2}}$ are the transmit powers of the first V2V vehicle and the second V2V vehicle, respectively. Furthermore, vectors $\mathbf{g}_{v_{1} r} \in \mathbb{R}^{L}$ and $\mathbf{g}_{v_{2} r} \in \mathbb{R}^{L}$ are the vectors of the channel gains between the $\mathrm{V} 2 \mathrm{~V}$ vehicles and $r$ in each of the predefined locations. The received signal power at $b$ from each predefined location of $r$ is denoted as $\mathbf{s}_{r b} \in \mathbb{R}^{L}$ and can be expressed as

$$
\mathbf{s}_{r b}=p_{r} \mathbf{g}_{r b} .
$$

where, the notation $p_{r}$ is the transmit power of the node $r$ and $\mathbf{g}_{r b} \in \mathbb{R}^{L}$ is the vector of the gains for the links between the locations of $r$ and the $b$. Finally, we define the interference signal powers received at the $\mathrm{V} 2 \mathrm{~V}$ vehicles from $r$ by $\mathbf{s}_{r v_{1}} \in$ $\mathbb{R}^{L}$ and $\mathbf{s}_{r v_{2}} \in \mathbb{R}^{L}$. These vectors are expressed as

$$
\begin{aligned}
& \mathbf{s}_{r v_{1}}=p_{r} \mathbf{g}_{r v_{1}}, \\
& \mathbf{s}_{r v_{2}}=p_{r} \mathbf{g}_{r v_{2}} .
\end{aligned}
$$

where, vectors $\mathbf{g}_{r v_{1}} \in \mathbb{R}^{L}$ and $\mathbf{g}_{r v_{2}} \in \mathbb{R}^{L}$ are the vectors of the channel gains between the $r$ in each of the predefined locations and $\mathrm{V} 2 \mathrm{~V}$ vehicles.

In order to be able to choose the best location from the set $\mathcal{L}$ we define a vector $\mathbf{e} \in \mathbb{R}^{L}$ whose entries must be all null except one entry which must be equal to one. The index of the nonzero entry in e demands that the set of coordinates with the same index from the location matrix $\mathbf{L}$ must be chosen for the relay to be located at. The vector e is expressed as

$$
\mathbf{e}^{T}=\left[\begin{array}{lllll}
0 & \ldots & 1 & \ldots & 0
\end{array}\right]
$$

By using notation e, we now rewrite the SINR expressions defined in (9), (10), and (11). The SINR of the link from $s$ to $r$ is expressed as

$$
\gamma_{s r}=\frac{\mathbf{e}^{T} \mathbf{s}_{s r}}{N_{0}+I_{r}+\sum_{j=1}^{2} \mathbf{e}^{T} \mathbf{s}_{r v_{j}}} .
$$

The SINR for each of the V2V links is calculated as

$$
\gamma_{v_{j}}=\frac{p_{v_{k}} g_{V 2 V}}{N_{0}+I_{v_{j}}+p_{s} g_{s v_{j}}+\mathbf{e}^{T} \mathbf{s}_{r v_{j i}}} \quad j, k \in\{1,2\}, j \neq k .
$$

Finally, we can calculate the SNR for the link from the relay to the destination:

$$
\gamma_{r d}=\frac{\mathbf{e}^{T} \mathbf{s}_{r d}}{N_{0}}
$$

Our goal is to find a location for the UAV to operate (from a given set of locations $\mathcal{L}$ ) such that the QoS of the V2V link, source to relay link, and relay to destination link is guaranteed. The QoS of these links can be guaranteed when the SINR of each link is greater than a predefined threshold. Hence, this

\begin{tabular}{l}
\hline Algorithm 1 UAV positioning algorithm \\
1: For a given topology: set SINR thresholds \\
2: Find SINRs for each location of $r$ \\
3: Approximate problem (23) by (24) \\
4: Solve (24) and find e \\
5: Find the index of the maximum value in e, and locate the UAV
\end{tabular}

design problem can be formulated as the following feasibility problem

minimize 0

$$
\begin{array}{ll}
\text { subject to } & \frac{\mathbf{e}^{T} \mathbf{s}_{s r}}{N_{0}+I_{r}+\sum_{i=1}^{2} \mathbf{e}^{T} \mathbf{s}_{\mathbf{r v}}} \geq \gamma_{1} \\
& \frac{p_{v_{j}} g_{V 2 V}}{N_{0}+I_{v_{i}}+p_{s} \mathbf{g}_{\mathbf{s} \mathbf{v}_{\mathbf{i}}}+\mathbf{e}^{\mathbf{T}} \mathbf{S}_{\mathbf{r} \mathbf{v}_{\mathbf{i}}}} \geq \gamma_{2}, i, j \in\{1,2\}, i \neq j \\
& \frac{\mathbf{e}^{T} \mathbf{s}_{r d}}{N_{0}} \geq \gamma_{3} \\
& \|\mathbf{e}\|_{0}=1 \\
& e_{k} \in\{0,1\}, \quad k=1, \ldots, L
\end{array}
$$

where the variable is e. Problem (23) is non-combinatorial and NP-hard, and it requires exponential complexity to find a global optimal solution [19]. Therefore, we have to rely on suboptimal methods to find an approximate solution to problem (23).

\section{B. Solution approach}

In the following, we approximate problem (23) as a convex optimization problem. A natural approximation of $\ell_{0}$ is its $\ell_{1}$-norm function. Hence, by replacing all the $\ell_{0}$ functions with their $\ell_{1}$-norm functions, we can write the approximated problem of (23) as follows

minimize 0

$$
\begin{array}{ll}
\text { subject to } & \frac{\mathbf{e}^{T} \mathbf{s}_{s r}}{N_{0}+I_{r}+\sum_{i=1}^{2} \mathbf{e}^{T} \mathbf{s}_{\mathbf{r} \mathbf{v}_{\mathbf{i}}}} \geq \gamma_{1} \\
& \frac{p_{v_{j}} g_{V 2 V}}{N_{0}+I_{v_{i}}+p_{s} \mathbf{g}_{\mathbf{s} \mathbf{v}_{\mathbf{i}}}+\mathbf{e}^{\mathbf{T}} \mathbf{S}_{\mathbf{r} \mathbf{v}_{\mathbf{i}}}} \geq \gamma_{2}, i, j \in\{1,2\}, i \neq j \\
& \frac{\mathbf{e}^{T} \mathbf{s}_{r d}}{N_{0}} \geq \gamma_{3} \\
& \|\mathbf{e}\|_{1} \leq 1 \\
& 0 \leq e_{k} \leq 1, \quad k=1, \ldots, L
\end{array}
$$

where the optimization variable is e. Note that the binary constraint in $(23 \mathrm{e})$ has been relaxed by introducing constraint (24e) in problem (24). This is a convex optimization problem and we can use any standard CVX solver to solve this problem. The proposed algorithm for solving the relay positioning problem is summarized in Algorithm 1.

\section{NumERICAL RESUlTS}

We consider a cross-road in which the source node and the $\mathrm{V} 2 \mathrm{~V}$ linked vehicles are located. Above this cross-road, we consider a square $114 \mathrm{~m} \times 144 \mathrm{~m}$ area with 400 predefined 
Table I: Environment parameters for A2G channel model.

\begin{tabular}{|l|c|c|c|c|}
\hline Environment & $\eta_{\operatorname{LoS}}$ & $\eta_{N \text { LoS }}$ & $\alpha$ & $\beta$ \\
\hline Suburban & 0.1 & 21 & 5.0188 & 0.3511 \\
\hline urban & 1 & 20 & 9.6101 & 0.1592 \\
\hline Dense urban & 1.6 & 23 & 11.9480 & 0.1359 \\
\hline High rise urban & 2.3 & 34 & 27.1562 & 0.1225 \\
\hline
\end{tabular}

Table II: Simulation parameters.

\begin{tabular}{|l|c|}
\hline Description & Value \\
\hline V2V transmit power $\left(P_{v_{1}}, P_{v_{2}}\right)$ & $0-1-0.4 \mathrm{~mW}$ \\
\hline Source transmit power $\left(P_{s}\right)$ & $0.5 \mathrm{~mW}$ \\
\hline Relay transmit power $\left(P_{r}\right)$ & $0.5 \mathrm{~mW}$ \\
\hline Carrier frequency $\left(f_{c}\right)$ & $2 \mathrm{MHz}$ \\
\hline Bandwidth $(B W)$ & $1 \mathrm{KHz}$ \\
\hline Number of the locations of the relay $(l)$ & 400 \\
\hline Reference path loss $\left(P L_{0}\right)$ & $63.9 \mathrm{dBm}$ \\
\hline Reference distance $\left(d_{0}\right)$ & $10 \mathrm{~m}$ \\
\hline Break-point distance $\left(d_{b}\right)$ & $161 \mathrm{~m}$ \\
\hline Path loss exponent $\left(n_{1}\right)$ & 1.81 \\
\hline Path loss exponent $\left(n_{2}\right)$ & 2.85 \\
\hline Reference distance $\left(d_{0}\right)$ & $10 \mathrm{~m}$ \\
\hline Noise power spectral density $\left(N_{0}\right)$ & $-170 \mathrm{dBm}$ \\
\hline BS antenna height $\left(h_{b}\right)$ & $30 \mathrm{~m}$ \\
\hline
\end{tabular}

locations for the relay. Locations are placed with $6 \mathrm{~m}$ distance from each other. We consider UAV communications in different environments with the carrier frequency of $2 \mathrm{GHz}$. The environment parameters are presented in Table I [4]. The BS is located at the coordinates of $(1000,1000)$ and the locations of the vehicles are randomly generated. We set the vehicles to be on either of the streets with a length of $1 \mathrm{~km}$. The distance between the V2V link is set to be $40 \mathrm{~m}$. For obtaining each of the points on the figures, we have run 500 simulations with different vehicular configurations. Each number on the $y$ axis presents the number of times that the problem is solved out of 500 times. In other words, $y$ axis shows how many times a proper location is found for the relay out of 500 times. Table II presents the simulation parameters.

Fig. 2 shows the number of feasible answers for the $\ell_{1}$ minimization problem compared to the number of the times that a UAV in a fixed location can satisfy the SINR requirement of the links in the system. The number of feasible answers of the problem increases by implementing the predefined locations for the relay due to the position options that the relay is provided with. These different locations offer a wide range of choices for the relay to choose to operate at. Since, each predefined location is assigned a set of SINRs values, the possibility that the relay can find a position that the SINRs set would satisfy the constraints increases. However, this does not happen when the UAV has a fixed location, and therefore, our proposed algorithm performs better in satisfying the SINR requirements of the system.

Fig. 3 shows the effect of different environments on the system where the same set of vehicle locations with an SINR constraint of $1 \mathrm{~dB}$ is considered. As it is depicted in Fig. 3 , in a suburban area the number of feasible answers to the problem are higher than an urban area and the feasible answers in an urban area are higher than that of a dense urban area. Moreover, there is a pattern in the results of all the environments and that is due to the fact that by increasing the altitude of the UAV the possibility of having a LoS link

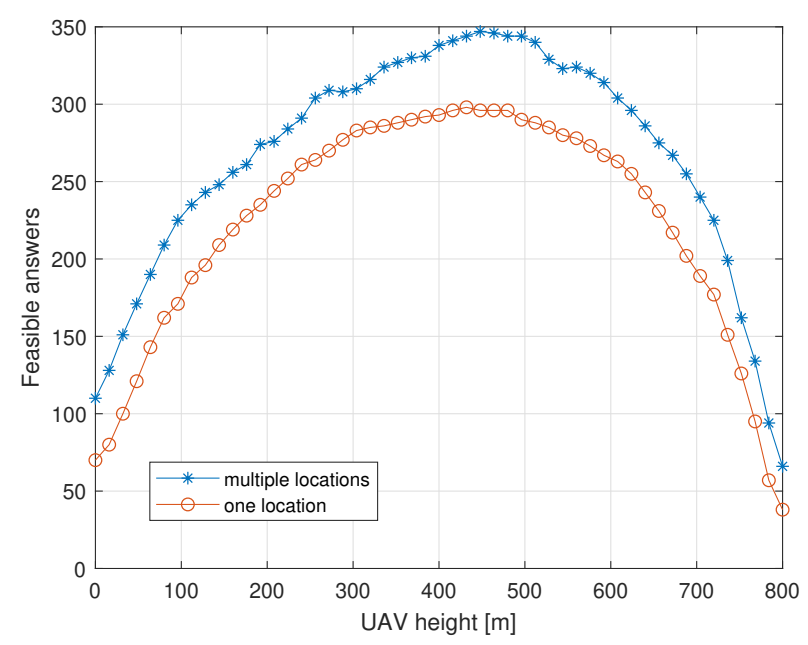

Figure 2: Number of feasible answers for a static UAV with one location compared to multiple locations.

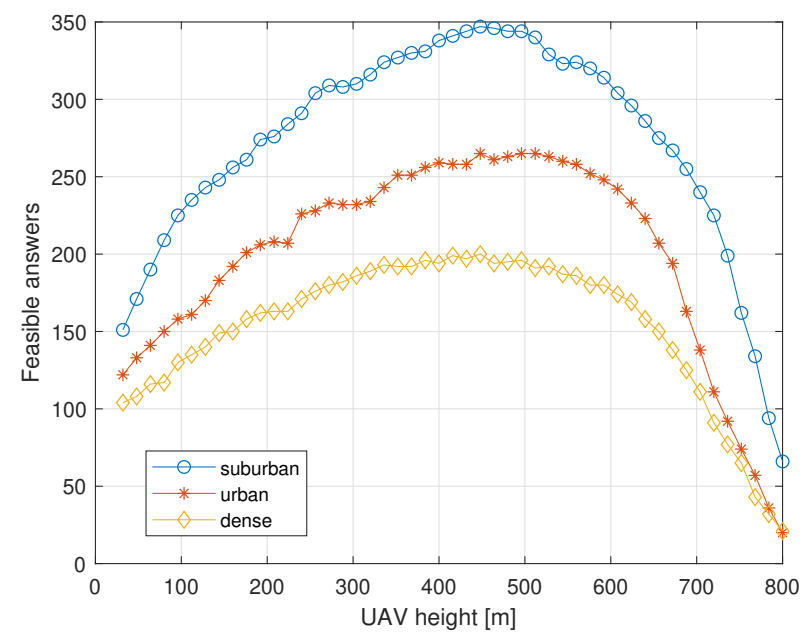

Figure 3: Number of feasible answers in different environments.

increases and we get more feasible answers. However, at some point this effect stops and the number of the feasible answers decrease due to the increase in the distance between the relay and the ground user which leads to a high pathloss.

Fig. 4 shows the number of feasible answers for the problem in an urban environment for different SINR constraints for the V2V link. The SINR constraint for the relay links are set to be $1 \mathrm{~dB}$, however, we change the V2V link SINR threshold from $1 \mathrm{~dB}$ to $4 \mathrm{~dB}$. As shown in Fig. 4 the number of feasible answers decrease by increasing the SINR threshold.

Fig. 5 shows the number of feasible answers for the problem in an urban environment while the transmit power of the source node and the relay are fixed at $0.5 \mathrm{~mW}$ but the transmit power of the $\mathrm{V} 2 \mathrm{~V}$ vehicles are changed from $0.1 \mathrm{~mW}$ to $0.4 \mathrm{~mW}$. As shown in Fig. 5, the number of feasible answers decreases as the $\mathrm{V} 2 \mathrm{~V}$ transmit power increases due to the excessive interference from the $\mathrm{V} 2 \mathrm{~V}$ on the transmission link from $s$ to $r$. Furthermore, by increasing the transmit power of the $s$ and $r$ the number of feasible answers increase as SINR of the 


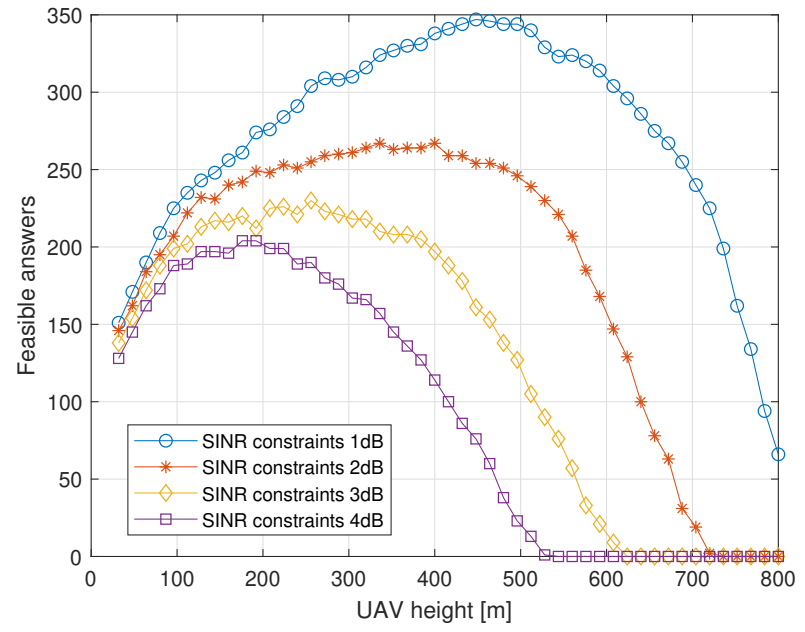

Figure 4: Number of feasible answers for different SINR constraints in a suburban environment.

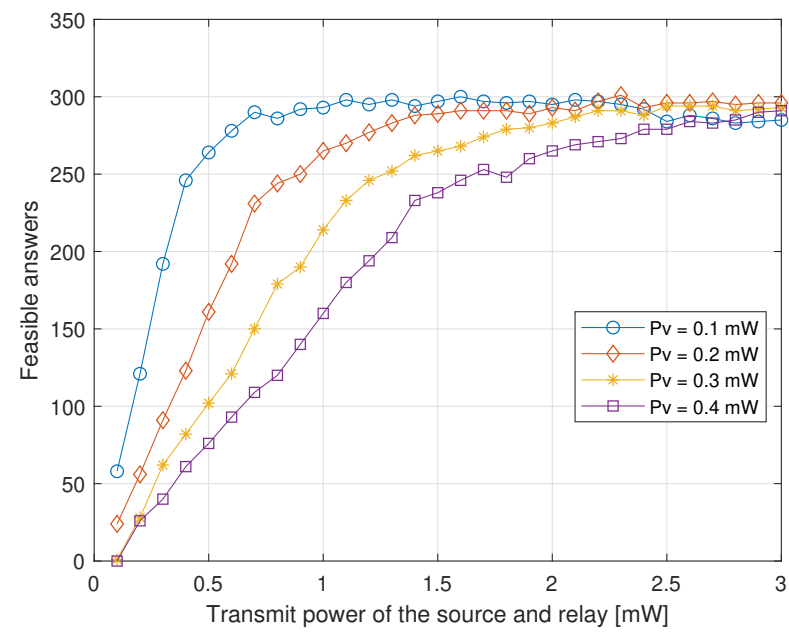

Figure 5: Number of feasible answers for different V2V transmit powers.

link from $s$ to $r$ improves.

\section{CONCLUSION}

In this paper, FD UAV relaying is proposed to increase wireless coverage in vehicular communication networks with an underlay V2V link. First, by using a set of predefined locations for the UAV relay, and, also by considering the locations of the vehicles on the ground, we have derived the SINRs for all the possible locations for the UAV. Second, to find the optimal location of the UAV, we have formulated an $\ell_{0}$-norm minimization problem. Finally, since the formulated problem is non-combinatorial and NP-hard, we have used an $\ell_{1}$-norm approximation for it, which results in a convex optimization problem. Simulation results have shown that by using the proposed method, the number of times that the UAV can find a location to satisfy the SINR requirements of all the links is $10 \%$ higher compared a baseline scenario in which the UAV has a fixed location.

\section{REFERENCES}

[1] A. Ferdowsi, U. Challita, W. Saad, and N. B. Mandayam, "Robust deep reinforcement learning for security and safety in autonomous vehicle systems," arXiv preprint arXiv:1805.00983, 2018.

[2] P. Belanovic, D. Valerio, A. Paier, T. Zemen, F. Ricciato, and C. F. Mecklenbrauker, "On wireless links for vehicle-to-infrastructure communications," IEEE Transactions on Vehicular Technology, vol. 59, no. 1, pp. 269-282, Jan 2010.

[3] S. Al-Sultan, M. M. Al-Doori, A. H. Al-Bayatti, and H. Zedan, "A comprehensive survey on vehicular ad hoc network," Journal of network and computer applications, vol. 37, pp. 380-392, 2014.

[4] A. Al-Hourani, S. Kandeepan, and A. Jamalipour, "Modeling air-toground path loss for low altitude platforms in urban environments," in 2014 IEEE Global Communications Conference, Dec 2014, pp. 28982904.

[5] M. Mozaffari, W. Saad, M. Bennis, and M. Debbah, "Mobile internet of things: Can uavs provide an energy-efficient mobile architecture?" in 2016 IEEE Global Communications Conference (GLOBECOM), Dec 2016, pp. 1-6.

[6] Y. Cai, F. R. Yu, J. Li, Y. Zhou, and L. Lamont, "Medium access control for unmanned aerial vehicle (UAV) ad-hoc networks with fullduplex radios and multipacket reception capability," IEEE Transactions on Vehicular Technology, vol. 62, no. 1, pp. 390-394, Jan 2013.

[7] M. Mozaffari, W. Saad, M. Bennis, and M. Debbah, "Unmanned aerial vehicle with underlaid device-to-device communications: Performance and tradeoffs," IEEE Transactions on Wireless Communications, vol. 15, no. 6, pp. 3949-3963, June 2016.

[8] M. Mozaffari, W. Saad, M. Bennis, Y.-H. Nam, and M. Debbah, "A tutorial on UAVs for wireless networks: Applications, challenges, and open problems," arXiv preprint arXiv:1803.00680, 2018.

[9] M. Mozaffari, W. Saad, M. Bennis, and M. Debbah, "Mobile unmanned aerial vehicles (UAVs) for energy-efficient internet of things communications," IEEE Transactions on Wireless Communications, vol. 16, no. 11, pp. 7574-7589, Nov 2017.

[10] A. Bazzi, B. M. Masini, A. Zanella, and I. Thibault, "On the performance of IEEE $802.11 \mathrm{p}$ and LTE-V2V for the cooperative awareness of connected vehicles," IEEE Transactions on Vehicular Technology, vol. 66, no. 11, pp. 10419-10432, 2017.

[11] B. Coll-Perales, M. Gruteser, and J. Gozalvez, "Evaluation of IEEE 802.11 ad for mmWave V2V communications," in Wireless Communications and Networking Conference Workshops (WCNCW), 2018 IEEE. IEEE, 2018, pp. 290-295.

[12] C. Campolo, A. Molinaro, A. O. Berthet, and A. Vinel, "Full-duplex radios for vehicular communications," IEEE Communications Magazine, vol. 55, no. 6, pp. 182-189, 2017.

[13] S. Ali, N. Rajatheva, and M. Latva-aho, "Full duplex device-to-device communication in cellular networks," in 2014 European Conference on Networks and Communications (EuCNC), June 2014, pp. 1-5.

[14] J. Choi, V. Va, N. Gonzalez-Prelcic, R. Daniels, C. R. Bhat, and R. W. Heath, "Millimeter-wave vehicular communication to support massive automotive sensing," IEEE Communications Magazine, vol. 54, no. 12, pp. 160-167, December 2016.

[15] F. Zeng, R. Zhang, X. Cheng, and L. Yang, "UAV-assisted data dissemination scheduling in VANETs," in 2018 IEEE International Conference on Communications (ICC), May 2018, pp. 1-6.

[16] T. Abbas, K. Sjöberg, J. Karedal, and F. Tufvesson, "A measurement based shadow fading model for vehicle-to-vehicle network simulations," International Journal of Antennas and Propagation, vol. 2015, 2015.

[17] A. Al-Hourani, S. Kandeepan, and S. Lardner, "Optimal lap altitude for maximum coverage," IEEE Wireless Communications Letters, vol. 3, no. 6, pp. 569-572, Dec 2014.

[18] M. Mozaffari, W. Saad, M. Bennis, and M. Debbah, "Drone small cells in the clouds: Design, deployment and performance analysis," in 2015 IEEE Global Communications Conference (GLOBECOM), Dec 2015, pp. 1-6.

[19] S. Boyd, " $\ell_{1}$-norm methods for convex-cardinality problems," 2007, available: http://www.stanford.edu/class/ee364b/lectures/11_slides.pdf. 